\title{
Aquacel Surgical Dressing Reduces the Rate of Acute PJI Following Total Joint Arthroplasty: A Case-Control Study.
}

Jenny Cai

The Rothman Institute at Thomas Jefferson University Hospital, Philadelphia, Pennsylvania

Joseph A Karam

The Rothman Institute at Thomas Jefferson University Hospital, Philadelphia, Pennsylvania

Javad Parvizi

The Rothman Institute at Thomas Jefferson University Hospital, Philadelphia, Pennsylvania

Eric B Smith

The Rothman Institute at Thomas Jefferson University Hospital, Philadelphia, Pennsylvania

Peter F. Sharkey

The Rothman Institute at Thomas Jefferson University Hospital, Philadelphia, Pennsylvania Follow this and additional works at: $\mathrm{https} / / / j \mathrm{jdc}$.jefferson.edu/rothman_institúte

Part of the Orthopedics Commons

Let us know how access to this document benefits you

\section{Recommended Citation}

Cai, Jenny; Karam, Joseph A; Parvizi, Javad; Smith, Eric B; and Sharkey, Peter F., "Aquacel Surgical Dressing Reduces the Rate of Acute PJI Following Total Joint Arthroplasty: A CaseControl Study." (2014). Rothman Institute Faculty Papers. Paper 47.

https://jdc.jefferson.edu/rothman_institute/47

This Article is brought to you for free and open access by the Jefferson Digital Commons. The Jefferson Digital Commons is a service of Thomas Jefferson University's Center for Teaching and Learning (CTL). The Commons is a showcase for Jefferson books and journals, peer-reviewed scholarly publications, unique historical collections from the University archives, and teaching tools. The Jefferson Digital Commons allows researchers and interested readers anywhere in the world to learn about and keep up to date with Jefferson scholarship. This article has been accepted for inclusion in Rothman Institute Faculty Papers by an authorized administrator of the Jefferson Digital Commons. For more information, please contact: JeffersonDigitalCommons@jefferson.edu. 
As submitted to:

The Journal of Arthroplasty And later published as:

4 Aquacel Surgical Dressing Reduces the Rate of Acute PJI Following

Volume 29, Issue 6, pages: 1098-100

DOI: $10.1016 /$ j.arth.2013.11.012

June 2014 


\section{Abstract}

An effort to prevent PJI has led to the development of antimicrobial dressings that

12 support wound healing. We sought to determine whether Aquacel Surgical dressing

13 independently reduces the rate of acute PJI following TJA. A single institution retrospective

14 chart review of 903 consecutive cases who received the Aquacel Surgical dressing and 875

15 consecutive cases who received standard gauze dressing was conducted to determine the

16 incidence of acute PJI (within 3 months). The incidence of acute PJI is $0.44 \%$ in the Aquacel

17 dressing group compared to $1.7 \%$ in the standard gauze dressing group $(\mathrm{P}=0.005)$. Multivariate analysis revealed that use of Aquacel dressing was an independent risk factor for reduction of PJI

19 (odds ratio of 0.165, 95\% confidence interval: 0.051-0.533). Aquacel Surgical dressing

20 significantly reduces the incidence of acute PJI.

21 
Periprosthetic joint infection (PJI) is one of the most dreaded complications that occur

24 after total joint arthroplasty (TJA). PJI is reported to occur in $1 \%-4 \%$ and $0.59 \%-2 \%$ of patients

25 who have undergone total knee and hip arthroplasty, respectively [1,2]. The infection causes physical, emotional, and financial strain to patients and their families as well as an immense

27 monetary burden to hospitals and our economy. The annual nationwide cost to control infection is approximately $\$ 250$ million. The cost of treating an individual PJI can be in excess of $\$ 50,000$ and if the offending organism is antibiotic resistant, i.e. MRSA, that cost can surpass $\$ 100,000$ $[3,4]$. Additionally, perioperative mortality associated with PJI can be 10 times greater than with 31 primary TJA $[5,6]$.

Eradication of infection often requires additional surgery and is distressful for both the treating physician and patient. While there are numerous possible causes for PJI, a few important

34 risk factors related to the wound itself have been identified including wound drainage and 35 superficial wound infections [7]. The traditional approach to wound care consists of a simple 36 dressing that could be removed after 1 or 2 days with the idea that the wound re-epithelializes 37 during that time and can then be left uncovered.[8]. Among efforts to prevent the occurrence of

41 infection rates [10]. weaved cellulose center that contours to the skin to eliminate dead space, absorbs exudates, 44 releases ionic silver to reduce microbial activity and supports wound healing [11]. Furthermore, 
45 the dressing seals the wound and prevents seepage of drainage beyond the dressing perimeter.

46 The objective of this study was to evaluate the effect of using this dressing on the occurrence of

47 acute PJI in patients undergoing TJA. We hypothesized that the Aquacel Ag Hydrofiber dressing

48 would support healing following surgery and possibly reduce the rate of acute PJI.

50 Methods

Prior to initiation of the study, institutional review board approval was obtained. Using

52 our computerized joint arthroplasty database, 950 consecutive patients who underwent primary

53 total hip or total knee arthroplasty between October 2010 and March 2012 and received the

54 Aquacel dressing were identified. A list of 950 consecutive patients who received standard

55 dressings and who were admitted to the hospital before implementing systematic use of the

56 Aquacel dressing from April 2007 to August 2010 was generated in a similar fashion. To allow

57 for consistency in the use of the new dressing, data from the initial 6 weeks when Aquacel

58 dressing was utilized were omitted. Exclusion criteria included hip hemiarthroplasty,

59 unicompartmental knee arthroplasty, TJA for fracture treatment, conversion TJA, and revision

60 TJA. Each case was reviewed to verify the exclusion criteria and collect demographic

61 information, medical comorbidities, intraoperative parameters and development of acute PJI. The

62 latter was defined as PJI occurring within 3 months of surgery based on the new definition

63 criteria established by the Musculoskeletal Infection Society [12]. After eliminating patients

64 based on the exclusion criteria, 903 patients with hip (392), knee (508) or hip and knee (3)

65 arthroplasties were retained in the Aquacel group and 875 patients with hip (376) or knee (499)

66 arthroplasty in the standard dressing group. The Aquacel dressing was applied on the surgical

67 site in sterile conditions in the operating room and kept in place for 5 days postoperatively. 
Standard dressing application consisted of sterile xeroform and gauze applied over the incision site in the operating room and wrapped in an ace bandage that remained in place for 2 days postoperatively.

In addition to the application of the Aquacel Surgical dressing, changes in clinical practice during the study period included the use of dual intravenous antibiotic prophylaxis with vancomycin and cefazolin (vs. cefazolin alone previously) and systematic irrigation with dilute betadine before wound closure. These changes occurred 9 and 4 months before the end of the study period respectively. A total of 37 patient-related and procedure-related risk factors were taken into account in a multivariate analysis model where the dependent variable was the development of acute PJI (Table 1). Statistical analyses were performed using R version 2.15.1 (R Foundation for Statistical Computing, Vienna, Austria).

\section{Results}

The prevalence of acute PJI was lower in the Aquacel group (0.44\%) compared to the standard dressing group (1.71\%). Bivariate analysis conducted with Fisher's test first showed this to be statistically significant $(\mathrm{P}=0.005)$. A backward stepwise logistic regression model retained 7 independent risk factors for PJI (of 37 variables), including the use of Aquacel dressing, with an independent odds ratio of 0.165 (95\% confidence interval: $0.051-0.533)$. Other independent significant risk factors for infection were as follows: older age, higher body mass index, smoking status, thyroid disease, liver disease and history of steroid treatment (Table 2). Notably, utilization of vancomycin prophylaxis and betadine irrigation were not shown to be significant independent protective factors for acute PJI. 
Discussion

PJI is a major healthcare concern with mental, physical and financial burden on affected patients. With projected exponential increases in its incidence and costs, and the predicted

94 reforms of healthcare reimbursement, prevention of this complication is gaining more

95 importance [13]. Wound healing problems and superficial surgical site infections have

96 consistently shown to be determining risk factors for the development of PJI [14,15]. Thus,

97 addressing these specific issues may prevent the occurrence of deep infection. The Aquacel

98 dressing has several features that could positively affect the wound environment: it sequesters

99 fluid to avoid tissue maceration, while at the same time releasing a gel that maintains a relatively

100 humid environment; it is also completely impermeable, preventing bacteria from entering the

101 wound site from the outside environment and maintaining hypoxia in the wound, which has been

102 shown to enhance healing and cellular immunity through the up-regulation of hypoxic-inducible

103 factors [16]. The addition of silver provides antimicrobial activity [17].

The use of the Aquacel dressing in TJA has previously been shown to create less need for

105 dressing changes, thus decreasing burden on healthcare personnel, diminishing superficial wound 106 problem, and avoiding delays in hospital discharge due to wound healing issues [18]. As the first 107 study to correlate Aquacel dressing with acute PJI, our results show that this dressing is an 108 effective measure to significantly reduce the occurrence of acute PJI after TJA, when compared 109 to standard dressings with gauze and tape. In our series, it independently reduced the rate of 110 acute PJI approximately sixfold.

The cost of one standard Aquacel dressing at our institution is $\$ 39.05$. The cost to treat a

112 PJI has been variably estimated to range from $\$ 50,000$ to over $\$ 100,000$ [13]. A standard taped

113 surgical gauze dressing costs approximately $\$ 5.00$. Therefore, the additional cost per case for an 
114 Aquacel dressing is about \$34.00. Infection after TJA has been reported to have an incidence 115 ranging from $1.0 \%$ to $2.0 \%$ [4]. In the United States., there are over 1,000,000 TKAs and THAs 116 performed annually [19]. Assuming the lowest cost $(\$ 50,000)$ of PJI treatment and the lower 117 incidence (1\%) of reported PJI, the annual costs to manage PJI in the United States likely exceed $118 \$ 500,000,000$. The cost of using an Aquacel dressing routinely in the United States after TJA 119 would add approximately $\$ 27,000,000$ in cost. If the reported fourfold reduction in PJI noted in 120 our study is accurate, the cost of PJI management in the United States could be reduced by at 121 approximately $\$ 375,000,000$ with use of an Aquacel dressing. Therefore, the additional cost 122 associated with routine use of the Aquacel dressing after TJA can be readily justified. We recognize several limitations to our study, such as, principally, its retrospective 124 design on a cohort of consecutive patients. Nonetheless, we were able to include a relatively 125 large number of subjects and all changes in practice, as well as potential confounding factors, 126 were taken into account in a multivariate model to ascertain the independent protective effect of 127 the Aquacel dressing. Our main concern was the confounding effect of intravenous vancomycin 128 prophylaxis and dilute betadine irrigation, two practices we implemented based on recent 129 supportive evidence in the literature [20,21]. However, these two factors did not reach a 130 significant effect on the development of PJI in our current study. This lack of significance is 131 possibly due to the limited number of subjects involved since these two practices were 132 introduced at our institution relatively late in the study period. Finally, our main outcome 133 measurement consisted of PJI occurring within 3 months of surgery. We elected to use the 3134 month minimum follow-up, in compliance with the recent recommendations of the Center for 135 Disease Control and Prevention, which uses this period to determine if an infection occurring 136 after surgery could be directly attributed to that procedure or not [22]. 
138 Aquacel Ag Surgical wound dressing with ionic silver significantly reduced the incidence of

139 acute PJI in our cohort of patients. Its systematic use suggests that it would be an effective

140 measure to prevent the occurrence of acute PJI following TJA and thus diminish the significant

141 healthcare costs and patient morbidity of PJI.

142 


\section{References}

1. Adeli B, Parvizi J. Strategies for the prevention of periprosthetic joint infection. J Bone Joint Surg 2012;94(11 Suppl A):42.

2. Bozic KJ, Ong K, Lau E, et al. Estimating risk in Medicare patients with THA: an electronic risk calculator for periprosthetic joint infection and mortality. Clin Orthop Relat Res 2013;471(2):574.

3. Berbari EF, Hanssen AD, Duffy MC, et al. Risk factors for prosthetic joint infection: case-control study. Clin Infect Dis 1998;27:1247.

4. Parvizi J, Pawasarat IM, Azzam KA, et al. Periprosthetic joint infection: the economic impact of methicillin-resistant infections. JOA 2010;25(6 Sup):103.

5. Cataldo MA, Petrosillo N, Cipriani M, et al. Prosthetic joint infection: recent developments in diagnosis and management. J Infect 2010;61(6):443.

6. Fisman DN, Reilly DT, Karchmer AW, et al. Clinical effectiveness and cost effectiveness of 2 management strategies for infected total hip arthroplasty in the elderly. Clin Infect Dis 2001;32(3):419.

7. Pulido L, Ghanem E, Joshi A, et al. Periprosthetic joint infection: the incidence, timing, and predisposing factors. Clin Orthop Relat Res 2008;466(7):1710.

8. Berg A, Fleischer S, Kuss O, et al. Timing of dress removal in the healing of surgical wounds by primary intention: quantitative systematic review protocol. J Adv Nurs 2011;68(2):264.

9. Vasconcelos A, Cavaco-Paulo Artur. Wound dressings for a proteolytic-rich environment. Appl Microbiol Biotechnol 2011;90(2):445.

10. Hutchinson JJ, McGuckin M. Occlusive dressings: a microbiologic and clinical review. Am J Infect Control 1990;18(4):257.

11. Hopper GP, Deakin AH, Crane EO, et al. Enhancing patient recovery following lower limb arthroplasty with a modern wound dressing: a prospective, comparative audit. J Wound Care 2012;21(4):200.

12. Parvizi J, Zmistowski B, Berbari EF, et al. New definition for periprosthetic joint infection: from the Workgroup of the Musculoskeletal Infection Society. Clin Orthop Relat Res 2011;469(11):2992.

13. Kurtz SM, Lau E, Watson H, et al. Economic burden of periprosthetic joint infection in the United States. J Arthroplasty 2012;27(8, Supplement):61. 
14. Pulido L, Ghanem E, Joshi A, et al. Periprosthetic joint infection: the incidence, timing, and predisposing factors. Clin Orthop Relat Res 2008;466(7):1710.

15. Berbari EF, Osmon DR, Lahr B, et al. The Mayo Joint Infection Risk Score: implication for surgical site infection reporting and risk stratification. Infect Control Hosp Epidemiol 2012;33(8):774.

16. Okumura CYM, Hollands A, Tran DN, et al. A new pharmacological agent (AKB-4924) stabilizes hypoxia inducible factor-1 (HIF-1) and increases skin innate

17. Jones SA, Bowler PG, Walker M, et al. Controlling wound bioburden with a novel silver-containing Hydrofiber dressing. Wound Repair Regen 2004;12(3):288.

18. Hopper GP, Deakin AH, Crane EO, et al. Enhancing patient recovery following lower limb arthroplasty with a modern wound dressing: a prospective, comparative audit. J Wound Care 2012 Apr;21(4):200.

19. Kurtz SM, Ong K, Lau E, et al. Projections of primary and revision hip and knee arthroplasty in the United States from 2005 to 2030. J Bone Joint Surg Am 2007;89: 780 .

20. Brown NM, Cipriano CA, Moric M, et al. Dilute betadine lavage before closure for the prevention of acute postoperative deep periprosthetic joint infection. J Arthroplasty 2012;27(1):27.

21. Smith EB, Wynne R, Joshi A, et al. Is it time to include vancomycin for routine perioperative antibiotic prophylaxis in total joint arthroplasty patients? J Arthroplasty 2012;27(8 Suppl):55. http://dx.doi.org/10.1016/j. arth.2012.03.040. [Epub 2012 May 17].

22. Mangram AJ, Horan TC, Pearson ML, et al. Guideline for prevention of surgical site infection, 1999. Centers for Disease Control and Prevention (CDC) Hospital Infection Control Practices Advisory Committee. Am J Infect Control 1999;27(2):97. 
224 Table 1 - List of patient-related and procedure-related factors included in the first step of the 225 logistic regression model

\begin{tabular}{|c|c|}
\hline Demographic factors & $\begin{array}{l}\text { Age } \\
\text { Gender } \\
\text { BMI } \\
\end{array}$ \\
\hline Procedure-related factors & $\begin{array}{l}\text { Joint } \\
\text { Bilateral procedure } \\
\text { OR time } \\
\text { Transfusion need } \\
\text { Type of anesthesia } \\
\text { Length of stay } \\
\text { Aquacel dressing } \\
\text { Dilute betadine irrigation }\end{array}$ \\
\hline$\underline{\text { Comorbidities }}$ & $\begin{array}{l}\text { Smoking status } \\
\text { Frequent alcohol drinking } \\
\text { History of MI } \\
\text { Congestive heart failure } \\
\text { Peripheral vascular disease } \\
\text { Cerebro-vascular disease } \\
\text { Dementia } \\
\text { Chronic pulmonary disease } \\
\text { Connective tissue disease } \\
\text { Coronary artery disease } \\
\text { Peptic ulcer disease } \\
\text { Liver disease } \\
\text { Diabetes mellitus } \\
\text { Chronic renal disease } \\
\text { Malignancy (history, active } \\
\text { disease or metastatic disease) } \\
\text { Rheumatoid disease } \\
\text { Hypertension } \\
\text { Dyslipidemia } \\
\text { Thyroid disease } \\
\text { Psychiatric disease } \\
\text { Anemia } \\
\text { Dysrythmia } \\
\text { History of DVT or PE } \\
\text { GERD } \\
\text { History of steroid treatment } \\
\text { ASA }\end{array}$ \\
\hline
\end{tabular}

228 ASA = American Society of Anaesthesiologistsphysical status classi-cation, BMI= Body Mass

229 Index, DVT = Deep VeinThrombosis, GERD = Gastro-EsophagealReux Disease, $\mathrm{MI}=$

230 Myocardial Infarction, $\mathrm{OR}=$ Operating Room, $\mathrm{PE}=$ PulmonaryEmbolism. 
Table 2 - Table 2. Factors included in the final logisticregression model with independent oddsratios and $95 \%$ confidence intervals

\begin{tabular}{|l|c|c|}
\hline & $\begin{array}{c}\text { Odds Ratio } \\
\text { Aquacel@ confidence interval) }\end{array}$ & p-value \\
\hline Age & $0.17(0.05-0.53)$ & 0.003 \\
\hline Body mass index & $1.09(1.03-1.14)$ & 0.002 \\
\hline Former Smoker & $1.10(1.03-1.19)$ & 0.006 \\
\hline Thyroid disease & $3.02(1.12-8.12)$ & 0.029 \\
\hline Liver disease & $3.71(1.42-9.67)$ & 0.007 \\
\hline History of systemic steroid treatment & $22.22(1.83-269.45)$ & 0.015 \\
\hline
\end{tabular}

